\title{
Helical Poly( $N$-propargylamide)s Derived from Lactic Acid: Effect of $O$-Acyl Groups on the Secondary Structures
}

\author{
By Fumio SANDA, ${ }^{*}$ Toru FUJII, Masashi SHIOTSUKI, and Toshio MASUDA ${ }^{\dagger, *}$
}

A series of optically active novel $O$-acylated $N$-propargyllactamide derivatives, $(S)$ - $\mathrm{HC} \equiv \mathrm{CCH}_{2} \mathrm{NHCOCH}\left(\mathrm{CH}_{3}\right) \mathrm{OCOC}_{2} \mathrm{H}_{5}$ (1), $(S)-\mathrm{HC} \equiv \mathrm{CCH}_{2} \mathrm{NHCOCH}\left(\mathrm{CH}_{3}\right) \mathrm{OCO}-n-\mathrm{C}_{4} \mathrm{H}_{9} \quad(\mathbf{2}),(S)-\mathrm{HC} \equiv \mathrm{CCH}_{2} \mathrm{NHCOCH}\left(\mathrm{CH}_{3}\right) \mathrm{OCOC}\left(\mathrm{CH}_{3}\right)_{3} \quad(\mathbf{3}), \quad(S)-\mathrm{HC} \equiv \mathrm{CCH}_{2}-$

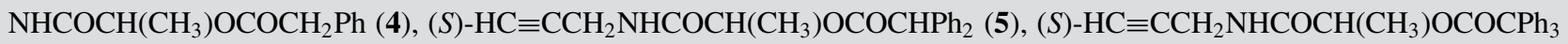
(6), $(S)-\mathrm{HC} \equiv \mathrm{CCH}_{2} \mathrm{NHCOCH}\left(\mathrm{CH}_{3}\right) \mathrm{OCO}-1,4-\mathrm{C}_{6} \mathrm{H}_{4}-n-\mathrm{C}_{6} \mathrm{H}_{13}$ (7), $(S)-\mathrm{HC} \equiv \mathrm{CCH}_{2} \mathrm{NHCOCH}\left(\mathrm{CH}_{3}\right) \mathrm{OCOCH}_{2} \mathrm{CH}_{2} \mathrm{Ph}(\mathbf{8})$, $(S)$ $\mathrm{HC} \equiv \mathrm{CCH}_{2} \mathrm{NHCOCH}\left(\mathrm{CH}_{3}\right) \mathrm{OCOCH} \mathrm{CH}_{2} \mathrm{CH}_{2} \mathrm{Ph}(\mathbf{9}),(S)-\mathrm{HC} \equiv \mathrm{CCH}_{2} \mathrm{NHCOCH}\left(\mathrm{CH}_{2} \mathrm{Ph}\right) \mathrm{OCOC}_{2} \mathrm{H}_{5}(\mathbf{1 0})$, and $(S)-\mathrm{HC} \equiv \mathrm{CCH}_{2}-$ $\mathrm{NHCOCH}\left(\mathrm{CH}_{2} \mathrm{Ph}\right) \mathrm{OCO}-1,4-\mathrm{C}_{6} \mathrm{H}_{4}-n-\mathrm{C}_{6} \mathrm{H}_{13}$ (11) were synthesized and polymerized with [(nbd) $\mathrm{RhCl}_{2}-\mathrm{Et}_{3} \mathrm{~N}$ as a catalyst to obtain the corresponding polymers with moderate molecular weights $\left(M_{\mathrm{n}}=10000-210000\right)$ in $58-95 \%$ yields. Polarimetric, CD, UV-vis, and IR spectroscopic studies revealed that the polymers took helical structures with predominantly one-handed screw sense stabilized by intramolecular hydrogen bonding between the amide groups in the side chains in various solvents. The bulkiness of the substituents affected the degree of one-handedness, tightness and stability of helical structure of the polymers. Poly(7) formed a liquid crystal.

KEY WORDS: Helix / Hydrogen Bond / Liquid Crystal / Optically Active Polymer / Polyacetylene /

Substituted polyacetylenes attract much attention due to unique and useful features such as photo- and electroluminescence properties, gas permeability, and helix formation. ${ }^{1}$ Monosubstituted acetylenes undergo polymerization with rhodium catalysts to give cis-stereoregular polyacetylenes. ${ }^{2}$ When appropriately chiral groups are substituted, polyaceytlenes form a helical structure with predominantly one-handed screw sense due to the rigid main chain. In the cases of poly(1alkyne)s, ${ }^{3}$ poly(phenylacetylene) derivatives, ${ }^{4}$ and poly(propiolic ester)s, ${ }^{5}$ bulky substituents stabilize the helical structure. Meanwhile, in the cases of poly( $N$-propargylamide $) s,{ }^{6}$ poly( $N$-propargylcarbamate)s, ${ }^{7}$ and poly(propargyl ester)s, ${ }^{8}$ amide and/or hydroxy groups stabilize the helical structure by intramolecular hydrogen bonding as well as steric repulsion between the side chains. These polymers reversibly form and deform intramolecular hydrogen bonds upon heating and solvent changes, leading to helix-helix or helix-coil transition.

We have recently reported the synthesis and polymerization of $N$-propargyllactamide derivatives; the formed polymers take a helical conformation under certain conditions, and they exhibit chiral recognition ability. ${ }^{9}$ The hydroxy group largely affects the helical structure. Namely, the polymers tend to form a helix in polar solvents, while they become random in a nonpolar medium $\left(\mathrm{CHCl}_{3}\right)$. This tendency is completely opposite to the case of poly( $N$-propargylamide)s bearing no hydroxy group reported so far. ${ }^{6}$ In polar solvents, the hydroxy groups presumably prevent solvent molecules from disturbing the formation of intramolecular hydrogen bonding between the amide groups. In $\mathrm{CHCl}_{3}$, in contrast, the hydroxy groups participate in hydrogen bonding between the amide groups of
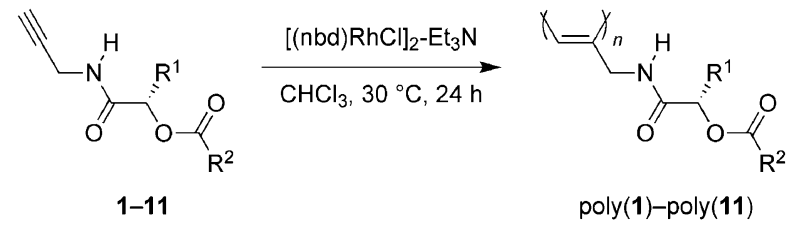

poly(1)-poly(11)

\begin{tabular}{cllcll}
\hline Monomer & $\mathrm{R}^{1}$ & \multicolumn{1}{c}{$\mathrm{R}^{2}$} & Monomer & \multicolumn{1}{c}{$\mathrm{R}^{1}$} & \multicolumn{1}{c}{$\mathrm{R}^{2}$} \\
\hline $\mathbf{1}$ & $\mathrm{CH}_{3}$ & $\mathrm{C}_{2} \mathrm{H}_{5}$ & $\mathbf{7}$ & $\mathrm{CH}_{3}$ & $1,4-\mathrm{C}_{6} \mathrm{H}_{4}-n-\mathrm{C}_{6} \mathrm{H}_{13}$ \\
$\mathbf{2}$ & $\mathrm{CH}_{3}$ & $n-\mathrm{C}_{4} \mathrm{H}_{9}$ & $\mathbf{8}$ & $\mathrm{CH}_{3}$ & $\mathrm{CH}_{2} \mathrm{CH}_{2} \mathrm{Ph}$ \\
$\mathbf{3}$ & $\mathrm{CH}_{3}$ & $\mathrm{C}\left(\mathrm{CH}_{3}\right)_{3}$ & $\mathbf{9}$ & $\mathrm{CH}_{3}$ & $\mathrm{CH}_{2} \mathrm{CH}_{2} \mathrm{CH}_{2} \mathrm{Ph}$ \\
$\mathbf{4}$ & $\mathrm{CH}_{3}$ & $\mathrm{CH}_{2} \mathrm{Ph}$ & $\mathbf{1 0}$ & $\mathrm{CH}_{2} \mathrm{Ph}$ & $\mathrm{C}_{2} \mathrm{H}_{5}$ \\
$\mathbf{5}$ & $\mathrm{CH}_{3}$ & $\mathrm{CHPh}_{2}$ & $\mathbf{1 1}$ & $\mathrm{CH}_{2} \mathrm{Ph}$ & $1,4-\mathrm{C}_{6} \mathrm{H}_{4}-n-\mathrm{C}_{6} \mathrm{H}_{13}$ \\
$\mathbf{6}$ & $\mathrm{CH}_{3}$ & $\mathrm{CPh}_{3}$ & & & \\
\hline
\end{tabular}

Scheme 1. Polymerization of 1-11.

the polymers, resulting in no one-handed helical structure. A wide variety of substituents can be incorporated using the hydroxy group at the chiral center, which possibly affects the helical structure. Although various poly( $N$-propargylamide)s have been reported, no poly $(N$-propargylamide) carrying $O$ acyl group at the chiral center has been reported yet. The purpose of this manuscript is examination of substituent effect on the helical properties of novel $O$-acylated $\mathrm{N}$-propargyl(phenyl)lactamide derivatives (Scheme 1).

\section{EXPERIMENTAL}

\section{Measurements}

Melting points (mp) were measured with a Yanaco micromelting point apparatus. IR spectra were obtained with a

Department of Polymer Chemistry, Graduate School of Engineering, Kyoto University, Katsura Campus, Kyoto 615-8510, Japan

†resent Address: Department of Environmental and Biotechnological Frontier Engineering, Faculty of Engineering, Fukui University of Technology, 3-61 Gakuen, Fukui 910-8505, Japan

*To whom correspondence should be addressed (E-mail: sanda@adv.polym.kyoto-u.ac.jp; masuda@fukui-ut.ac.jp). 
JASCO FT/IR-4100 spectrophotometer. ${ }^{1} \mathrm{H}$ and ${ }^{13} \mathrm{C}$ NMR $\left({ }^{1} \mathrm{H}\right.$ : $400 \mathrm{MHz},{ }^{13} \mathrm{C}: 100 \mathrm{MHz}$ ) spectra were recorded on a JEOL EX-400 spectrometer. Elemental analyses were conducted at the Micro Analytical Center of Kyoto University. Number- and weight-average molecular weights $\left(M_{\mathrm{n}}\right.$ and $\left.M_{\mathrm{w}}\right)$ of polymers were estimated by GPC (Shodex KF-850L columns) eluted with THF by use of a polystyrene calibration. CD and UV-vis spectra were recorded on a JASCO J-820 spectropolarimeter.

\section{Materials}

All the reagents were used as purchased without purification unless otherwise stated. $\mathrm{CHCl}_{3}$ and $\mathrm{Et}_{3} \mathrm{~N}$ used for polymerization were distilled by the standard procedure before use.

Synthesis of 1-3, 5, and 7-9: Typical Procedure. A solution of propargylamine $(0.69 \mathrm{~mL}, 10.0 \mathrm{mmol})$ and $4-(4,6-\mathrm{di}-$ methoxy-1,3,5-triazin-2-yl)-4-methylmorpholinium chloride (TRIAZIMOCH, Tokuyama, $15 \mathrm{wt} \% \mathrm{H}_{2} \mathrm{O}, 3.25 \mathrm{~g}, 10.0 \mathrm{mmol}$ ) in THF $(100 \mathrm{~mL})$ was added to a solution of $\mathrm{L}-(+)$-lactic acid $(1.6 \mathrm{~g}, 10.0 \mathrm{mmol})$ at room temperature. The resulting solution was stirred at room temperature overnight. White precipitate formed was filtered off, and the filtrate was concentrated on a rotary evaporator. The residue and diphenylacetic acid was dissolved in $\mathrm{CH}_{2} \mathrm{Cl}_{2}(100 \mathrm{~mL})$. 4-(Dimethylamino)pyridine $(0.12 \mathrm{~g}, 1.0 \mathrm{mmol})$ and 1-[3-(dimethylamino)propyl]-3-ethylcarbodiimide hydrochloride $(\mathrm{EDC} \cdot \mathrm{HCl}, 2.11 \mathrm{~g}, 11.0 \mathrm{mmol})$ were added to the solution at $0{ }^{\circ} \mathrm{C}$, and the resulting mixture was stirred at room temperature overnight. After that, the mixture was subsequently washed with $1 \mathrm{M} \mathrm{HCl}$ aq., saturated $\mathrm{NaHCO}_{3}$ aq., and $\mathrm{NaCl}$ aq. twice, and then dried over anhydrous $\mathrm{MgSO}_{4} \cdot \mathrm{CH}_{2} \mathrm{Cl}_{2}$ was distilled off on a rotary evaporator. Then, the residue was purified by silica gel column chromatography eluted with hexane/ethyl acetate to obtain $\mathbf{5}$. Synthesis of 4. A solution of propargylamine $(1.38 \mathrm{~mL}, 20.0$ mmol) and TRIAZIMOCH (15 wt \% $\left.\mathrm{H}_{2} \mathrm{O}, 6.50 \mathrm{~g}, 20.0 \mathrm{mmol}\right)$ in THF $(100 \mathrm{~mL})$ was added to a solution of L- $(+)$-lactic acid $(3.2 \mathrm{~g}, 20.0 \mathrm{mmol})$ at room temperature. The resulting solution was stirred at room temperature overnight. White precipitate formed was filtered off, and the filtrate was concentrated on a rotary evaporator. Diethyl ether $(200 \mathrm{~mL})$ was added to the residue, and then pyridine $(1.73 \mathrm{~mL}, 20 \mathrm{mmol})$ and phenylacetyl chloride $(2.64 \mathrm{~mL}, 20 \mathrm{mmol})$ were added to the solution sequentially. The resulting mixture was stirred at $0{ }^{\circ} \mathrm{C}$ for $2 \mathrm{~h}$, and white precipitate formed was filtered off. The filtrate was subsequently washed with $1 \mathrm{M} \mathrm{HCl}$ aq., saturated $\mathrm{NaHCO}_{3}$ aq., and $\mathrm{NaCl}$ aq. twice, and then dried over anhydrous $\mathrm{MgSO}_{4}$. It was concentrated on a rotary evaporator, and the residue was purified by silica gel column chromatography eluted with hexane/ethyl acetate to obtain 4 .

Synthesis of 6. Triphenylacetic acid $(2.88 \mathrm{~g}, 10 \mathrm{mmol})$ was added to thionyl chloride $(7.33 \mathrm{~mL}, 100 \mathrm{mmol})$, and the resulting solution was heated under reflux for $3 \mathrm{~h}$. The residual thionyl chloride was removed on a rotary evaporator to obtain crude triphenylacetyl chloride. A solution of propargylamine $(1.38 \mathrm{~mL}, 20.0 \mathrm{mmol})$ and TRIAZIMOCH (15 wt \% $\mathrm{H}_{2} \mathrm{O}$, $6.50 \mathrm{~g}, 20.0 \mathrm{mmol})$ in THF $(100 \mathrm{~mL})$ was added to a solution of $\mathrm{L}-(+)$-lactic acid $(3.2 \mathrm{~g}, 20.0 \mathrm{mmol})$ at room temperature.
The resulting solution was stirred at room temperature overnight. White precipitate formed was filtered off, and the filtrate was concentrated on a rotary evaporator. THF/DMF $(3: 1$, volume ratio, $80 \mathrm{~mL}$ ) was added to the residue, and then crude triphenylacetyl chloride ( $c a .10 \mathrm{mmol}$ ) was added to the solution. $\mathrm{NaH}(0.48 \mathrm{~g}, 20 \mathrm{mmol})$ was slowly added to the mixture, and it was stirred at room temperature for $1 \mathrm{~d}$. $\mathrm{MeOH}$ $(30 \mathrm{~mL})$ was added to the mixture to quench the reaction. The solvents were removed under reduced pressure, and then $\mathrm{CHCl}_{3}$ was added to the residue. The solution was subsequently washed with $1 \mathrm{M} \mathrm{HCl}$ aq., saturated $\mathrm{NaHCO}_{3}$ aq., and $\mathrm{NaCl}$ aq. twice, dried over anhydrous $\mathrm{MgSO}_{4}$, and concentrated on a rotary evaporator. The residue was purified by silica gel column chromatography eluted with hexane/ethyl acetate to obtain 6.

Synthesis of 10 and 11. Monomers 10 and 11 were synthesized from propargylamine, L-(-)-3-phenyllactic acid, and the corresponding carboxylic acids in a manner similar to $\mathbf{5}$.

Synthesis of $\mathbf{5}^{\prime}$. A mixture of L-lactic acid $(9.0 \mathrm{~g}, 0.1 \mathrm{~mol})$, propargyl alcohol $(23.3 \mathrm{~g}, 0.4 \mathrm{~mol})$, sulfuric acid $(6 \mathrm{M}$, $0.09 \mathrm{~mL})$, and benzene $(5.5 \mathrm{~mL})$ was heated under reflux for $4 \mathrm{~h}$. The resulting solution was neutralized with $\mathrm{CH}_{3} \mathrm{COOK}$, and distilled under reduced pressure to obtain $(S)$-propargyl 2-hydroxypropionate. $\mathrm{EDC} \cdot \mathrm{HCl}(2.11 \mathrm{~g}, 11.0 \mathrm{mmol})$ and 4(dimethylamino)pyridine $(0.12 \mathrm{~g}, 1.0 \mathrm{mmol})$ were added to a solution of $(S)$-propargyl 2-hydroxypropionate (1.28 g, 10.0 $\mathrm{mmol})$ and diphenylacetic acid in $\mathrm{CH}_{2} \mathrm{Cl}_{2}(50 \mathrm{~mL})$ at $0{ }^{\circ} \mathrm{C}$, and the resulting mixture was stirred at room temperature overnight. After that, the mixture was subsequently washed with $1 \mathrm{M} \mathrm{HCl}$ aq., saturated $\mathrm{NaHCO}_{3}$ aq., and $\mathrm{NaCl}$ aq. twice, and then dried over anhydrous $\mathrm{MgSO}_{4}$. It was concentrated on a rotary evaporator, and the residue was purified by silica gel column chromatography eluted with hexane/ethyl acetate to obtain $\mathbf{5}^{\prime}$.

\section{Data of the Monomers}

1. Yield $=42 \%$ (colorless oil). $[\alpha]_{\mathrm{D}}=-24.4^{\circ} \quad(c=0.10$ $\left.\mathrm{g} \cdot \mathrm{dL}^{-1}, \mathrm{CHCl}_{3}\right)$. IR $\left(\mathrm{CHCl}_{3}\right): 3384(\mathrm{H}-\mathrm{C} \equiv), 2920,2912,2122$ $(\mathrm{C} \equiv \mathrm{C}), 1646(\mathrm{C}=\mathrm{O}), 1543(\mathrm{~N}-\mathrm{H}) \mathrm{cm}^{-1} \cdot{ }^{1} \mathrm{H}$ NMR $\left(\mathrm{CDCl}_{3}\right)$ : $\delta=1.17\left(\mathrm{CH}_{2} \mathrm{CH}_{3}, \mathrm{t}, J=7.6 \mathrm{~Hz}, 3 \mathrm{H}\right), 1.46\left(\mathrm{CH}_{3}, \mathrm{~d}, J=\right.$ $6.8 \mathrm{~Hz}, 3 \mathrm{H}), 2.24(\mathrm{C} \equiv \mathrm{CH}, \mathrm{s}, 1 \mathrm{H}), 2.41\left(\mathrm{CH}_{2} \mathrm{CH}_{3}, \mathrm{q}, J=7.2\right.$ $\mathrm{Hz}, 2 \mathrm{H}), 4.08\left(\mathrm{C} \equiv \mathrm{CCH}_{2}, \mathrm{~m}, 2 \mathrm{H}\right), 5.23\left(\mathrm{CHCH}_{3}, \mathrm{q}, J=6.8 \mathrm{~Hz}\right.$, $1 \mathrm{H}), 6.28(\mathrm{NH}$, br s, $1 \mathrm{H}) .{ }^{13} \mathrm{C} \mathrm{NMR}\left(\mathrm{CDCl}_{3}\right): \delta=9.0,17.8$, 27.6, 29.0, 70.3, 72.0, 77.3, 170.0, 172.7. $\mathrm{C}_{9} \mathrm{H}_{13} \mathrm{NO}_{3}$ : Calcd. $\mathrm{C}$ 59.00, H 7.15, N 7.65; Found: C 58.63, H 7.01, N 7.50.

2. Yield $=30 \%$ (colorless oil). $[\alpha]_{\mathrm{D}}=-26.7^{\circ}(c=0.10$ $\left.\mathrm{g} \cdot \mathrm{dL}^{-1}, \mathrm{CHCl}_{3}\right)$. IR $\left(\mathrm{CHCl}_{3}\right): 3310(\mathrm{H}-\mathrm{C} \equiv), 3020,2112$ $(\mathrm{C} \equiv \mathrm{C}), 1748(\mathrm{C}=\mathrm{O}), 1686(\mathrm{C}=\mathrm{O}), 1521(\mathrm{~N}-\mathrm{H}), 1219,1046$, $930783, \quad 738, \quad 627 \mathrm{~cm}^{-1} .{ }^{1} \mathrm{H} \mathrm{NMR} \quad\left(\mathrm{CDCl}_{3}\right): \quad \delta=0.88$ $\left(\mathrm{CH}_{2} \mathrm{CH}_{2} \mathrm{CH}_{2} \mathrm{CH}_{3}, \mathrm{t}, \mathrm{J}=6.8 \mathrm{~Hz}, 3 \mathrm{H}\right), 1.30\left(\mathrm{CH}_{2} \mathrm{CH}_{2} \mathrm{CH}_{2} \mathrm{CH}_{3}\right.$, $\mathrm{q}, J=6.8 \mathrm{~Hz}, 2 \mathrm{H}), 1.43\left(\mathrm{CH}_{2} \mathrm{CH}_{2} \mathrm{CH}_{2} \mathrm{CH}_{3}, \mathrm{~m}, 2 \mathrm{H}\right), 1.58$ $\left(\mathrm{CH}_{3}, \mathrm{~m}, 3 \mathrm{H}\right), 2.20(\mathrm{C} \equiv \mathrm{CH}, \mathrm{s}, 1 \mathrm{H}), 2.35\left(\mathrm{CH}_{2} \mathrm{CH}_{3}, \mathrm{q}, J=7.2\right.$ $\mathrm{Hz}, 2 \mathrm{H}), 4.02\left(\mathrm{C} \equiv \mathrm{CCH}_{2}, \mathrm{~m}, 2 \mathrm{H}\right), 5.19\left(\mathrm{CHCH}_{3}, \mathrm{q}, J=6.8 \mathrm{~Hz}\right.$, $1 \mathrm{H}), 6.40(\mathrm{~N} H$, br s, $1 \mathrm{H}) .{ }^{13} \mathrm{C} \mathrm{NMR}\left(\mathrm{CDCl}_{3}\right): \delta=13.6,17.7$, 22.1, 26.8, 28.9, 33.9, 70.0, 71.8, 78.9, 170.1, 172.1; $\mathrm{C}_{11} \mathrm{H}_{17^{-}}$ $\mathrm{NO}_{3}$ : Calcd. C 62.54, H 8.11, N 6.63; Found: C 62.10, H 8.56, N 6.02. 
3. Yield $=15 \%$ (colorless oil). $[\alpha]_{\mathrm{D}}=-30.1^{\circ} \quad(c=0.10$ $\left.\mathrm{g} \cdot \mathrm{dL}^{-1}, \mathrm{CHCl}_{3}\right)$. IR $\left(\mathrm{CHCl}_{3}\right): 3382(\mathrm{H}-\mathrm{C} \equiv), 2920,2912,2114$ $(\mathrm{C} \equiv \mathrm{C}), \quad 1745 \quad(\mathrm{C}=\mathrm{O}), \quad 1664 \quad(\mathrm{C}=\mathrm{O}), 1520(\mathrm{~N}-\mathrm{H}) \quad \mathrm{cm}^{-1}$. ${ }^{1} \mathrm{H} \mathrm{NMR}\left(\mathrm{CDCl}_{3}\right): \delta=1.24\left[\mathrm{C}\left(\mathrm{CH}_{3}\right)_{3}, \mathrm{~s}, 9 \mathrm{H}\right], 1.43\left(\mathrm{CH}_{3}, \mathrm{~d}\right.$, $J=6.8 \mathrm{~Hz}, 3 \mathrm{H}), 2.24(\mathrm{C} \equiv \mathrm{CH}, \mathrm{s}, 1 \mathrm{H}), 4.02\left(\mathrm{C} \equiv \mathrm{CCH}_{2}, \mathrm{~m}, 2 \mathrm{H}\right)$, $5.21\left(\mathrm{CHCH}_{3}, \mathrm{q}, J=6.8 \mathrm{~Hz}, 1 \mathrm{H}\right), 6.16(\mathrm{NH}$, br s, $1 \mathrm{H})$. ${ }^{13} \mathrm{C} \mathrm{NMR}\left(\mathrm{CDCl}_{3}\right): \delta=17.5,27.0,29.1,38.7,70.1,72.0,78.9$, 170.2, 176.7. $\mathrm{C}_{11} \mathrm{H}_{17} \mathrm{NO}_{3}$ : Calcd. C 62.54, H 8.11, N 6.63; Found: C 61.74, H 8.20, N 6.54.

4. Yield $=28 \%$ (white solid). $\mathrm{Mp}=60.0-61.2^{\circ} \mathrm{C}$. $[\alpha]_{\mathrm{D}}=$ $+48.5^{\circ}\left(c=0.10 \mathrm{~g} \cdot \mathrm{dL}^{-1}, \mathrm{CHCl}_{3}\right)$. IR $\left(\mathrm{CHCl}_{3}\right): 3303(\mathrm{H}-\mathrm{C} \equiv)$, 3019, $2136(\mathrm{C} \equiv \mathrm{C}), 1743(\mathrm{C}=\mathrm{O}), 1686(\mathrm{C}=\mathrm{O}), 1524(\mathrm{~N}-\mathrm{H})$, 1213, 734, $669 \mathrm{~cm}^{-1} .{ }^{1} \mathrm{H} \mathrm{NMR}\left(\mathrm{CDCl}_{3}\right): \delta=1.38\left(\mathrm{CH}_{3}, \mathrm{~m}\right.$, $3 \mathrm{H}), 2.14(\mathrm{C} \equiv \mathrm{CH}, \mathrm{s}, 1 \mathrm{H}), 3.52\left(\mathrm{CH}_{2} \mathrm{Ph}, \mathrm{m}, 2 \mathrm{H}\right), 3.85$ $\left(\mathrm{C} \equiv \mathrm{CCH}_{2}, \mathrm{~m}, 2 \mathrm{H}\right), 5.16\left(\mathrm{CHCH}_{3}, \mathrm{q}, J=6.8 \mathrm{~Hz}, 1 \mathrm{H}\right), 5.84$ $\left(\mathrm{NH}\right.$, br s, 1H), 7.19-7.32 (Ph, m, 5H). ${ }^{13} \mathrm{C} \mathrm{NMR}\left(\mathrm{CDCl}_{3}\right)$ : $\delta=17.7,28.9,41.6,70.6,71.8,78.8,172.5,129.0133 .4,169.6$, 169.8. $\mathrm{C}_{14} \mathrm{H}_{15} \mathrm{NO}_{3}$ : Calcd. C 68.56, H 6.16, N 5.71; Found: C 68.61, H 6.17, N 5.60.

5. Yield $=47 \%$ (white solid). $\mathrm{Mp}=100.2-101.0^{\circ} \mathrm{C} .[\alpha]_{\mathrm{D}}=$ $+28.4^{\circ}\left(c=0.10 \mathrm{~g} \cdot \mathrm{dL}^{-1}, \mathrm{CHCl}_{3}\right)$. IR $\left(\mathrm{CHCl}_{3}\right): 3684,3622$, 3426, $3308(\mathrm{H}-\mathrm{C} \equiv), 3019,2122(\mathrm{C} \equiv \mathrm{C}), 1742(\mathrm{C}=\mathrm{O}), 1676$ $(\mathrm{C}=\mathrm{O}), 1521(\mathrm{~N}-\mathrm{H}), 1423,1218,1046,929,779,669$, $627 \mathrm{~cm}^{-1} .{ }^{1} \mathrm{H} \mathrm{NMR}\left(\mathrm{CDCl}_{3}\right): \delta=1.48\left(\mathrm{CHCH}_{3}, \mathrm{~d}, J=6.8\right.$ $\mathrm{Hz}, 3 \mathrm{H}), 2.18(\mathrm{C} \equiv \mathrm{CH}, \mathrm{s}, 1 \mathrm{H}), 3.87\left(\mathrm{C} \equiv \mathrm{CCH}_{2}, \mathrm{~m}, 2 \mathrm{H}\right), 5.11$ $[\mathrm{CHPh}, \mathrm{s}, 1 \mathrm{H}], 5.34\left(\mathrm{CHCH}_{3}, \mathrm{~d}, J=6.8 \mathrm{~Hz}, 1 \mathrm{H}\right), 5.81(\mathrm{NH}$, br s, $1 \mathrm{H}), 7.25-7.40\left(\mathrm{Ph}_{2}\right.$, br s, $\left.10 \mathrm{H}\right) .{ }^{13} \mathrm{C} \mathrm{NMR}\left(\mathrm{CDCl}_{3}\right)$ : $\delta=17.6,28.5,57.2,71.1,71.6,78.8,127.7,128.5,129.5$, 137.6, 170.0, 170.5. $\mathrm{C}_{20} \mathrm{H}_{19} \mathrm{NO}_{3}$ : Calcd. C 74.75, H 5.96, N 4.36; Found: C 73.97, H 5.98, N 4.31.

6. Yield $=44 \%$ (white solid). $\mathrm{Mp}=126.2-127.0^{\circ} \mathrm{C}$. $[\alpha]_{\mathrm{D}}=$ $+28.4^{\circ}\left(c=0.10 \mathrm{~g} \cdot \mathrm{dL}^{-1}, \mathrm{CHCl}_{3}\right)$. IR $\left(\mathrm{CHCl}_{3}\right): 3417,3308$ $(\mathrm{H}-\mathrm{C} \equiv), 3011,2122(\mathrm{C} \equiv \mathrm{C}), 1742(\mathrm{C}=\mathrm{O}), 1681(\mathrm{C}=\mathrm{O}), 1522$ $(\mathrm{N}-\mathrm{H}), 1495,1448,1174,1084,1036,709,667,615 \mathrm{~cm}^{-1}$. ${ }^{1} \mathrm{H} \mathrm{NMR}\left(\mathrm{CDCl}_{3}\right): \delta=1.50\left(\mathrm{CHCH}_{3}, \mathrm{~d}, J=6.8 \mathrm{~Hz}, 3 \mathrm{H}\right), 2.17$ $(\mathrm{C} \equiv \mathrm{CH}, \mathrm{s}, 1 \mathrm{H}), 3.77\left(\mathrm{C} \equiv \mathrm{CCH}_{2}, \mathrm{~m}, 2 \mathrm{H}\right), 5.38\left(\mathrm{CHCH}_{3}, \mathrm{~d}\right.$, $J=6.8 \mathrm{~Hz}, 1 \mathrm{H}), 5.80(\mathrm{NH}$, br s, $1 \mathrm{H}), 7.25-7.33\left(\mathrm{Ph}_{3}\right.$, br s, 15H). ${ }^{13} \mathrm{C} \mathrm{NMR}\left(\mathrm{CDCl}_{3}\right): \delta=17.4,28.5,67.2,71.2,71.5$, 78.9, 127.2, 128.1, 129.9, 142.2, 169.6, 171.4. $\mathrm{C}_{26} \mathrm{H}_{23} \mathrm{NO}_{3}$ : Calcd. C 78.57, H 5.83, N 3.52; Found: C 78.16, H 6.06, N 3.53. 7. Yield $=38 \%$ (white solid). $\mathrm{Mp}=75.0-75.6^{\circ} \mathrm{C} .[\alpha]_{\mathrm{D}}=$ $+39.8^{\circ}\left(c=0.10 \mathrm{~g} \cdot \mathrm{dL}^{-1}, \mathrm{CHCl}_{3}\right)$. IR $\left(\mathrm{CHCl}_{3}\right): 3455,3308$ $(\mathrm{H}-\mathrm{C} \equiv), 3019,2126(\mathrm{C} \equiv \mathrm{C}), 1723(\mathrm{C}=\mathrm{O}), 1686(\mathrm{C}=\mathrm{O}), 1519$ $(\mathrm{N}-\mathrm{H}), 1213,1102,759,669 \mathrm{~cm}^{-1} .{ }^{1} \mathrm{H} \mathrm{NMR}\left(\mathrm{CDCl}_{3}\right): \delta=$ $0.86\left(\mathrm{CH}_{2} \mathrm{CH}_{2} \mathrm{CH}_{2} \mathrm{CH}_{2} \mathrm{CH}_{2} \mathrm{CH}_{3}\right.$, s, 3H), $1.26\left(\mathrm{CH}_{2} \mathrm{CH}_{2} \mathrm{CH}_{2}-\right.$ $\left.\mathrm{CH}_{2} \mathrm{CH}_{2} \mathrm{CH}_{3}, \quad \mathrm{~m}, \quad 6 \mathrm{H}\right), \quad 1.58 \quad\left(\mathrm{CH}_{2} \mathrm{CH}_{2} \mathrm{CH}_{2} \mathrm{CH}_{2} \mathrm{CH}_{2} \mathrm{CH}_{3}\right.$, $\left.\mathrm{CHCH}_{3}, \mathrm{~m}, 5 \mathrm{H}\right), 2.22(\mathrm{C} \equiv \mathrm{CH}, \mathrm{s}, 1 \mathrm{H}), 2.66\left(\mathrm{CH}_{2} \mathrm{CH}_{2} \mathrm{CH}_{2}-\right.$ $\left.\mathrm{CH}_{2} \mathrm{CH}_{2} \mathrm{CH}_{3}, \mathrm{t}, J=8.0 \mathrm{~Hz}, 2 \mathrm{H}\right), 4.06\left(\mathrm{C} \equiv \mathrm{CCH}_{2}, \mathrm{~s}, 2 \mathrm{H}\right), 5.47$ $\left(\mathrm{CHCH}_{3}, \mathrm{~d}, J=6.8 \mathrm{~Hz}, 1 \mathrm{H}\right), 6.37(\mathrm{NH}$, br s, $1 \mathrm{H}), 7.26(\mathrm{Ar}, \mathrm{d}$, $J=8.4 \mathrm{~Hz}, 2 \mathrm{H}), 7.96(\mathrm{Ar}, \mathrm{d}, J=8.0 \mathrm{~Hz}, 2 \mathrm{H}) .{ }^{13} \mathrm{C} \mathrm{NMR}$ $\left(\mathrm{CDCl}_{3}\right): \delta=17.1,17.6,22.6,28.9,29.1,31.1,31.6,36.0$, 70.6, 71.9, 79.0, 126.6, 128.7, 129.8, 149.5, 165.1, 170.0. $\mathrm{C}_{19} \mathrm{H}_{25} \mathrm{NO}_{3}$ : Calcd. C 72.35, H 7.99, N 4.44; Found: C 71.52, H 8.09, N 4.86 .

8. Yield $=67 \%$ (white solid). $\mathrm{Mp}=57.0-58.0{ }^{\circ} \mathrm{C} .[\alpha]_{\mathrm{D}}=$ $-7.8^{\circ}\left(c=0.10 \mathrm{~g} \cdot \mathrm{dL}^{-1}, \mathrm{CHCl}_{3}\right)$. IR $\left(\mathrm{CHCl}_{3}\right): 3308(\mathrm{H}-\mathrm{C} \equiv)$,
3019, $2136(\mathrm{C} \equiv \mathrm{C}), 1745(\mathrm{C}=\mathrm{O}), 1684(\mathrm{C}=\mathrm{O}), 1520(\mathrm{~N}-\mathrm{H})$, 1213, 734, $669 \mathrm{~cm}^{-1} .{ }^{1} \mathrm{H} \mathrm{NMR}\left(\mathrm{CDCl}_{3}\right): \delta=1.38\left(\mathrm{CH}_{3}, \mathrm{~d}\right.$, $J=6.8 \mathrm{~Hz}, 3 \mathrm{H}), 2.23(\mathrm{C} \equiv \mathrm{CH}, \mathrm{t}, J=2.4 \mathrm{~Hz}, 1 \mathrm{H}), 2.77$ $\left(\mathrm{CH}_{2} \mathrm{CH}_{2} \mathrm{Ph}, \mathrm{m}, 2 \mathrm{H}\right), 3.00 \quad\left(\mathrm{CH}_{2} \mathrm{CH}_{2} \mathrm{Ph}, \mathrm{m}, 2 \mathrm{H}\right), 4.03$ $\left(\mathrm{C} \equiv \mathrm{CCH}_{2}, \mathrm{~m}, 2 \mathrm{H}\right), 5.20\left(\mathrm{CHCH}_{3}, \mathrm{~m}, 1 \mathrm{H}\right), 5.94(\mathrm{NH}$, br s, 1H), 7.21-7.33 (Ph, m, 5H). ${ }^{13} \mathrm{C} \mathrm{NMR}\left(\mathrm{CDCl}_{3}\right): \delta=17.7$, 28.9, 30.9, 35.7, 70.4, 71.7, 79.1, 126.5128.2, 128.7, 139.9, 169.8, 171.0. $\mathrm{C}_{15} \mathrm{H}_{17} \mathrm{NO}_{3}$ : Calcd. C 69.48, H 6.61, N 5.40; Found: C 69.37, H 6.45, N 5.21.

9. Yield $=52 \%$ (white solid). $\mathrm{Mp}=57.0-58.0^{\circ} \mathrm{C} .[\alpha]_{\mathrm{D}}=$ $-14.6^{\circ}\left(c=0.10 \mathrm{~g} \cdot \mathrm{dL}^{-1}, \mathrm{CHCl}_{3}\right)$. IR $\left(\mathrm{CHCl}_{3}\right): 3450,3308$, $(\mathrm{H}-\mathrm{C} \equiv), 3019,2115(\mathrm{C} \equiv \mathrm{C}), 1744(\mathrm{C}=\mathrm{O}), 1685(\mathrm{C}=\mathrm{O}), 1520$ $(\mathrm{N}-\mathrm{H}), 1213,1046,929,739,669 \mathrm{~cm}^{-1} .{ }^{1} \mathrm{H} \mathrm{NMR}\left(\mathrm{CDCl}_{3}\right)$ : $\delta=1.48\left(\mathrm{CH}_{3}, \mathrm{~d}, J=6.8 \mathrm{~Hz}, 3 \mathrm{H}\right), 2.00\left(\mathrm{CH}_{2} \mathrm{CH}_{2} \mathrm{CH}_{2} \mathrm{Ph}, \mathrm{m}\right.$, $2 \mathrm{H}), 2.04(\mathrm{C} \equiv \mathrm{CH}, \mathrm{s}, 1 \mathrm{H}), 2.41\left(\mathrm{CH}_{2} \mathrm{CH}_{2} \mathrm{CH}_{2} \mathrm{Ph}, \mathrm{m}, 2 \mathrm{H}\right), 2.67$ $\left(\mathrm{CH}_{2} \mathrm{CH}_{2} \mathrm{CH}_{2} \mathrm{Ph}, \mathrm{t}, J=6.8 \mathrm{~Hz}, 2 \mathrm{H}\right), 4.06\left(\mathrm{C} \equiv \mathrm{CCH}_{2}, \mathrm{~m}, 2 \mathrm{H}\right)$, $5.21\left(\mathrm{CHCH}_{3}, \mathrm{~d}, J=6.8 \mathrm{~Hz}, 1 \mathrm{H}\right), 6.42(\mathrm{NH}$, br s, $1 \mathrm{H}), 7.17-$ $7.31(\mathrm{Ph}, \mathrm{m}, 5 \mathrm{H}) .{ }^{13} \mathrm{C} \mathrm{NMR}\left(\mathrm{CDCl}_{3}\right): \delta=17.7,26.2,28.9$, 33.4, 34.9, 70.2, 71.8, 80.0, 126.0, 128.3, 140.9, 170.0, 171.0. $\mathrm{C}_{16} \mathrm{H}_{19} \mathrm{NO}_{3}$ : Calcd. C 70.31, H 7.01, N 5.12; Found: C 70.73, H 6.95, N 5.01.

10. Yield $=53 \%$ (white solid). $\mathrm{Mp}=69.2-69.4{ }^{\circ} \mathrm{C} .[\alpha]_{\mathrm{D}}=$ $-6.4^{\circ}\left(c=0.10 \mathrm{~g} \cdot \mathrm{dL}^{-1}, \mathrm{CHCl}_{3}\right)$. IR $\left(\mathrm{CHCl}_{3}\right): 3447,3308(\mathrm{H}-$ $\mathrm{C} \equiv), 3019,2122(\mathrm{C} \equiv \mathrm{C}), 1748(\mathrm{C}=\mathrm{O}), 1684(\mathrm{C}=\mathrm{O}), 1521(\mathrm{~N}-$ $\mathrm{H}), 1218,1046,929,767,669 \mathrm{~cm}^{-1} .{ }^{1} \mathrm{H} \mathrm{NMR}\left(\mathrm{CDCl}_{3}\right): \delta=$ $1.09\left(\mathrm{CH}_{2} \mathrm{CH}_{3}, \mathrm{t}, J=7.6 \mathrm{~Hz}, 3 \mathrm{H}\right), 2.21(\mathrm{C} \equiv \mathrm{CH}, \mathrm{s}, 1 \mathrm{H}), 2.32$ $\left(\mathrm{CH}_{2} \mathrm{CH}_{3}, \mathrm{q}, J=6.8 \mathrm{~Hz}, 2 \mathrm{H}\right), 3.10-3.16(\mathrm{CHHPh}, \mathrm{dd}, J=$ $14.0,7.2 \mathrm{~Hz}, 1 \mathrm{H}), 3.20-3.25(\mathrm{CH} H \mathrm{Ph}, \mathrm{dd}, J=14.0,4.8 \mathrm{~Hz}$, $1 \mathrm{H}), 4.00\left(\mathrm{C} \equiv \mathrm{CCH}_{2}, \mathrm{~m}, 2 \mathrm{H}\right), 5.41\left[\mathrm{CHCH}_{2} \mathrm{Ph}, \mathrm{dd}, J=6.8\right.$, $4.8 \mathrm{~Hz}, 1 \mathrm{H}], 6.23(\mathrm{NH}$, br s, $1 \mathrm{H}), 7.15-7.28(\mathrm{Ph}$, br s, 5H). ${ }^{13} \mathrm{C} \mathrm{NMR}\left(\mathrm{CDCl}_{3}\right): \delta=8.8,27.4,28.8,37.6,71.8,73.8,78.8$, 126.8, 128.3, 129.5, 135.6, 168.8, 172.7. $\mathrm{C}_{15} \mathrm{H}_{17} \mathrm{NO}_{3}$ : Calcd. $\mathrm{C}$ 69.48, H 6.61, N 5.40; Found: C 69.48, H 6.54, N 5.40.

11. Yield $=39 \%$ (white solid). $\mathrm{Mp}=95.0-96.0^{\circ} \mathrm{C}$. $[\alpha]_{\mathrm{D}}=$ $-9.3^{\circ}\left(c=0.10 \mathrm{~g} \cdot \mathrm{dL}^{-1}, \mathrm{CHCl}_{3}\right)$. IR $\left(\mathrm{CHCl}_{3}\right): 3447,3308(\mathrm{H}-$ $\mathrm{C} \equiv), 3019,2122(\mathrm{C} \equiv \mathrm{C}), 1731(\mathrm{C}=\mathrm{O}), 1684(\mathrm{C}=\mathrm{O}), 1521(\mathrm{~N}-$ $\mathrm{H}), 1215,1046,929,765,669 \mathrm{~cm}^{-1} .{ }^{1} \mathrm{H} \mathrm{NMR}\left(\mathrm{CDCl}_{3}\right): \delta=$ $0.89\left(\mathrm{CH}_{2} \mathrm{CH}_{2} \mathrm{CH}_{2} \mathrm{CH}_{2} \mathrm{CH}_{2} \mathrm{CH}_{3}, \mathrm{t}, \quad J=6.8 \mathrm{~Hz}, 3 \mathrm{H}\right), \quad 1.31$ $\left(\mathrm{CH}_{2} \mathrm{CH}_{2} \mathrm{CH}_{2} \mathrm{CH}_{2} \mathrm{CH}_{2} \mathrm{CH}_{3}, \quad \mathrm{~m}, 6 \mathrm{H}\right), 1.62 \quad\left(\mathrm{CH}_{2} \mathrm{CH}_{2} \mathrm{CH}_{2}-\right.$ $\left.\mathrm{CH}_{2} \mathrm{CH}_{2} \mathrm{CH}_{3}, \mathrm{~m}, 2 \mathrm{H}\right), 2.15(\mathrm{C} \equiv \mathrm{CH}, \mathrm{s}, 1 \mathrm{H}), 2.67\left(\mathrm{CH}_{2} \mathrm{CH}_{2}-\right.$ $\left.\mathrm{CH}_{2} \mathrm{CH}_{2} \mathrm{CH}_{2} \mathrm{CH}_{3}, \mathrm{~m}, 2 \mathrm{H}\right), 3.30-3.32\left(\mathrm{CH}_{2} \mathrm{Ph}, \mathrm{m}, 2 \mathrm{H}\right), 4.00$ $\left(\mathrm{C} \equiv \mathrm{CCH}_{2}, \mathrm{~m}, 2 \mathrm{H}\right), 5.64\left[\mathrm{CH}(\mathrm{OH}) \mathrm{CH}_{2} \mathrm{Ar}, \mathrm{m}, 1 \mathrm{H}\right], 6.05(\mathrm{NH}$, br s, 1H), 7.22-7.25 (ArH, br s, 7H), 7.85-7.88 ( $\mathrm{ArH}$, br s, 2H). ${ }^{13} \mathrm{C} \mathrm{NMR}\left(\mathrm{CDCl}_{3}\right): \delta=14.1,22.6,28.9,29.0,31.1,31.7,36.1$, 37.8, 71.8, 74.3, 78.8, 126.4, 126.9, 128.4, 128.6, 128.7, 129.7, 135.7, 149.5, 165.0, 168.9. $\mathrm{C}_{25} \mathrm{H}_{29} \mathrm{NO}_{3}$ : Calcd. C 71.06, $\mathrm{H}$ 7.37, N 4.87; Found: C 71.73, H 7.45, N 4.89.

5'. Yield $=63 \%($ colorless oil $) . \quad[\alpha]_{\mathrm{D}}=+46.4^{\circ} \quad(c=0.10$ $\left.\mathrm{g} \cdot \mathrm{dL}^{-1}, \mathrm{CHCl}_{3}\right)$. IR $\left(\mathrm{CHCl}_{3}\right): 3307(\mathrm{H}-\mathrm{C} \equiv), 3019,2125$ $(\mathrm{C} \equiv \mathrm{C}), 1743(\mathrm{C}=\mathrm{O}), 1496,1453,1096,780,666 \mathrm{~cm}^{-1}$. ${ }^{1} \mathrm{H} \mathrm{NMR}\left(\mathrm{CDCl}_{3}\right): \delta=1.50\left(\mathrm{CHCH}_{3}, \mathrm{~d}, J=6.4 \mathrm{~Hz}, 3 \mathrm{H}\right)$, $2.48(\mathrm{C} \equiv \mathrm{CH}, \mathrm{s}, 1 \mathrm{H}), 4.70\left(\mathrm{C} \equiv \mathrm{CCH}_{2}, \mathrm{~m}, 2 \mathrm{H}\right), 5.12\left(\mathrm{CHPh}_{2}, \mathrm{~s}\right.$, $1 \mathrm{H}), 5.15\left(\mathrm{CHCH}_{3}, \mathrm{q}, J=6.8,14.0 \mathrm{~Hz}, 1 \mathrm{H}\right), 7.18-7.60\left(\mathrm{Ph}_{2}\right.$, br s, $10 \mathrm{H}) .{ }^{13} \mathrm{C} \mathrm{NMR}\left(\mathrm{CDCl}_{3}\right): \delta=16.7,52.7,56.5,69.0,75.4$, 76.9, 127.2, 128.4, 128.6, 138.0, 169.6, 171.8. $\mathrm{C}_{20} \mathrm{H}_{18} \mathrm{O}_{4}$ : Calcd. C 74.52, H 5.63; Found: C 74.36, H 5.88. 


\section{Polymerization Procedure}

$\mathrm{A} \mathrm{CHCl}_{3}$ solution of a monomer $\left([\mathrm{M}]_{0}=1.0 \mathrm{M}\right)$ was added to a $\mathrm{CHCl}_{3}$ solution of $[(\mathrm{nbd}) \mathrm{RhCl}]_{2}\left([\mathrm{M}]_{0} /[\mathrm{Rh}]=100\right)$ and $\mathrm{Et}_{3} \mathrm{~N}\left([\mathrm{Rh}] /\left[\mathrm{Et}_{3} \mathrm{~N}\right]=1 / 10\right)$ in a glass tube equipped with a three-way stopcock under dry nitrogen, and the solution was kept at $30^{\circ} \mathrm{C}$ for $24 \mathrm{~h}$. After that, the reaction mixture was poured into a large amount of $\mathrm{MeOH}$ to precipitate a polymer. The resulting polymer was separated by filtration and dried under reduced pressure.

\section{Spectroscopic Data of the Polymers}

Poly(1). ${ }^{1} \mathrm{H}$ NMR $\left(\mathrm{CDCl}_{3}\right): \delta=0.96-1.22\left(\mathrm{CH}_{2} \mathrm{CH}_{3}\right), 1.22-$ $1.56\left(\mathrm{CHCH}_{3}\right), 2.22-2.60\left(\mathrm{CH}_{2} \mathrm{CH}_{3}\right), 3.52-4.40\left(\mathrm{C}=\mathrm{CCH}_{2}\right)$, 4.80-5.20 $\left(\mathrm{CHCH}_{3}\right), 5.80-6.30(\mathrm{C}=\mathrm{CH}), 7.60-8.18(\mathrm{NH})$.

Poly(2). IR (KBr): 3301, 3021, 2401, 1738, 1664, 1526, 1424, $1219,1046,930,669 \mathrm{~cm}^{-1} .{ }^{1} \mathrm{H}$ NMR $\left(\mathrm{CDCl}_{3}\right): \delta=0.60-0.98$ $\left(\mathrm{CH}_{2} \mathrm{CH}_{2} \mathrm{CH}_{2} \mathrm{CH}_{3}\right)$, 0.98-1.70 $\left(\mathrm{CH}_{2} \mathrm{CH}_{2} \mathrm{CH}_{2} \mathrm{CH}_{3}, \mathrm{CHCH}_{3}\right)$, 2.10-2.36 $\left(\mathrm{CH}_{2} \mathrm{CH}_{2} \mathrm{CH}_{2} \mathrm{CH}_{3}\right), 3.60-4.10\left(\mathrm{C}=\mathrm{CCH}_{2}\right)$, 4.76$5.20\left(\mathrm{CHCH}_{3}\right), 5.80-6.20(\mathrm{C}=\mathrm{CH}), 7.70-8.36(\mathrm{NH})$.

Poly(3). IR $\left(\mathrm{CHCl}_{3}\right)$ : 3305, 3021, 2401, 1738, 1646, 1526, 1424, 1219, 1046, 930, $669 \mathrm{~cm}^{-1}$. ${ }^{1} \mathrm{H} \mathrm{NMR}\left(\mathrm{CDCl}_{3}\right): \delta=$ 1.30-1.80 $\left[\mathrm{CHCH}_{3}, \mathrm{C}\left(\mathrm{CH}_{3}\right)_{3}\right], 1.62-1.80\left(\mathrm{CHCH}_{3}\right), 2.70-4.60$ $\left(\mathrm{C}=\mathrm{CCH}_{2}\right), 4.60-5.22\left(\mathrm{CHCH}_{3}\right), 5.50-6.50(\mathrm{C}=\mathrm{CH}), 7.60-$ $8.30(\mathrm{NH})$.

Poly(4). IR $\left(\mathrm{CHCl}_{3}\right): 3281,3090,1739,1659,1539,1261$, 1096, $908 \mathrm{~cm}^{-1} .{ }^{1} \mathrm{H} \mathrm{NMR}\left(\mathrm{CDCl}_{3}\right): \delta=0.96-1.52\left(\mathrm{CHCH}_{3}\right)$, 3.56-3.80 $\quad\left(\mathrm{CH}_{2} \mathrm{Ph}\right), \quad 3.80-4.40 \quad\left(\mathrm{C}=\mathrm{CCH}_{2}\right), \quad 4.80-5.24$ $\left(\mathrm{CHCH}_{3}\right), \quad 5.82-6.20 \quad(\mathrm{C}=\mathrm{CH}), \quad 6.86-7.40 \quad(\mathrm{Ph}), \quad 7.56-8.10$ (NH).

Poly(5). IR $\left(\mathrm{CHCl}_{3}\right): 3275,1740,1659,1542,1213,1151$, $1095,1213,1031,669 \mathrm{~cm}^{-1} .{ }^{1} \mathrm{H}$ NMR $\left(\mathrm{CDCl}_{3}\right): \delta=0.80-1.68$ $\left(\mathrm{CHCH}_{3}\right), 2.60-4.60\left(\mathrm{C}=\mathrm{CCH}_{2}\right), 4.70-5.50\left[\mathrm{CHCH}_{3}, \mathrm{CHPh}_{2}\right]$, 5.60-6.36 $(\mathrm{C}=\mathrm{CH}), 6.70-7.58\left(\mathrm{Ph}_{2}\right), 7.60-8.40(\mathrm{NH})$.

Poly(6). IR $\left(\mathrm{CHCl}_{3}\right): 3407,3279,1731,1664,1547,1221$, $1093 \mathrm{~cm}^{-1} .{ }^{1} \mathrm{H}$ NMR $\left(\mathrm{CDCl}_{3}\right): \delta=0.40-1.42\left(\mathrm{CHCH}_{3}\right), 2.70$ $4.20\left(\mathrm{C}=\mathrm{CCH}_{2}\right), 4.30-5.25\left(\mathrm{CHCH}_{3}\right), 5.50-6.30(\mathrm{C}=\mathrm{CH})$, 6.30-8.00 $\left(\mathrm{Ph}_{3}, \mathrm{NH}\right)$.

Poly(7). IR $\left(\mathrm{CHCl}_{3}\right): 3296,3019,2931,2857,1722,1656$, 1557, 1274, 1213, 1114, $669 \mathrm{~cm}^{-1} .{ }^{1} \mathrm{H} \mathrm{NMR}\left(\mathrm{CDCl}_{3}\right): \delta=$ 0.86-0.90 $\left(\mathrm{CH}_{2} \mathrm{CH}_{2} \mathrm{CH}_{2} \mathrm{CH}_{2} \mathrm{CH}_{2} \mathrm{CH}_{3}\right), 1.08-1.32\left(\mathrm{CH}_{2} \mathrm{CH}_{2}-\right.$ $\left.\mathrm{CH}_{2} \mathrm{CH}_{2} \mathrm{CH}_{2} \mathrm{CH}_{3}\right), \quad 1.32-1.70 \quad\left(\mathrm{CHCH}_{3}, \mathrm{CH}_{2} \mathrm{CH}_{2} \mathrm{CH}_{2} \mathrm{CH}_{2}-\right.$ $\left.\mathrm{CH}_{2} \mathrm{CH}_{3}\right)$, 2.30-2.70 $\left(\mathrm{CH}_{2} \mathrm{CH}_{2} \mathrm{CH}_{2} \mathrm{CH}_{2} \mathrm{CH}_{2} \mathrm{CH}_{3}\right), 3.70-3.92$ $\left(\mathrm{C}=\mathrm{CCH}_{2}\right), 4.20-4.60\left(\mathrm{CHCH}_{3}\right), 6.12-6.56(\mathrm{C}=\mathrm{CH}), 6.80-$ 7.12 (Ar), 7.50-7.90 (Ar), 7.90-8.26 (NH).

Poly(8). IR $\left(\mathrm{CHCl}_{3}\right): 3277,3019,1739,1541,1212,732$, $669 \mathrm{~cm}^{-1} .{ }^{1} \mathrm{H}$ NMR $\left(\mathrm{CDCl}_{3}\right): \delta=0.96-1.60\left(\mathrm{CHCH}_{3}\right), 2.20-$ $2.82 \quad\left(\mathrm{CH}_{2} \mathrm{CH}_{2} \mathrm{Ph}\right), \quad 2.82-3.10 \quad\left(\mathrm{CH}_{2} \mathrm{CH}_{2} \mathrm{Ph}\right), \quad 3.10-4.60$ $\left(\mathrm{C}=\mathrm{CCH}_{2}\right), 4.70-5.30\left(\mathrm{CHCH}_{3}\right), 5.70-6.40(\mathrm{C}=\mathrm{CH}), 6.80-$ $7.32(\mathrm{ArH}), 7.50-8.40(\mathrm{NH})$.

Poly(9). IR $\left(\mathrm{CHCl}_{3}\right): 3283,3019,1738,1662,1531,1213$, $669 \mathrm{~cm}^{-1} .{ }^{1} \mathrm{H} \mathrm{NMR}\left(\mathrm{CDCl}_{3}\right): \delta=1.10-1.60\left(\mathrm{CHCH}_{3}\right), 1.85-$ $2.10\left(\mathrm{CH}_{2} \mathrm{CH}_{2} \mathrm{CH}_{2} \mathrm{Ph}\right), 2.15-2.48\left(\mathrm{CH}_{2} \mathrm{CH}_{2} \mathrm{CH}_{2} \mathrm{Ph}\right), 2.48-2.80$ $\left(\mathrm{CH}_{2} \mathrm{CH}_{2} \mathrm{CH}_{2} \mathrm{Ph}\right), 3.50-4.26\left(\mathrm{C}=\mathrm{CCH}_{2}\right), 4.85-5.28\left(\mathrm{CHCH}_{3}\right)$, 5.80-6.20 $(\mathrm{C}=\mathrm{CH}), 7.00-7.32(\mathrm{Ph}), 7.58-8.00(\mathrm{NH})$.

Poly(10). IR $\left(\mathrm{CHCl}_{3}\right): 3242,3091,1741,1669,1541,1210$, 1180, 772, $669 \mathrm{~cm}^{-1}$. ${ }^{1} \mathrm{H} \mathrm{NMR}\left(\mathrm{CDCl}_{3}\right): \quad \delta=0.50-1.00$
$\left(\mathrm{CH}_{2} \mathrm{CH}_{3}\right), 1.80-2.40\left(\mathrm{CH}_{2} \mathrm{CH}_{3}\right), 2.55-3.40\left(\mathrm{CH}_{2} \mathrm{Ph}\right), 4.10-$ $4.50\left(\mathrm{C}=\mathrm{CCH}_{2}\right), 5.00-5.60\left(\mathrm{CHCH}_{2} \mathrm{Ph}\right), 5.80-6.15(\mathrm{C}=\mathrm{CH})$, 6.70-7.60 (Ph), 8.80-9.50 (NH).

Poly(11). IR $\left(\mathrm{CHCl}_{3}\right): 3270,3019,2929,1723,1656,1542$, $1260,1212,1113,669 \mathrm{~cm}^{-1} .{ }^{1} \mathrm{H} \mathrm{NMR}\left(\mathrm{CDCl}_{3}\right): \delta=0.60-1.00$ $\left(\mathrm{CH}_{2} \mathrm{CH}_{2} \mathrm{CH}_{2} \mathrm{CH}_{2} \mathrm{CH}_{2} \mathrm{CH}_{3}\right), \quad 1.00-1.40 \quad\left(\mathrm{CH}_{2} \mathrm{CH}_{2} \mathrm{CH}_{2} \mathrm{CH}_{2}-\right.$ $\left.\mathrm{CH}_{2} \mathrm{CH}_{3}\right), 2.10-2.80\left(\mathrm{CH}_{2} \mathrm{CH}_{2} \mathrm{CH}_{2} \mathrm{CH}_{2} \mathrm{CH}_{2} \mathrm{CH}_{3}\right), 2.90-3.96$ $\left(\mathrm{CH}_{2} \mathrm{Ph}\right), 4.12-4.50\left(\mathrm{C}=\mathrm{CCH}_{2}\right), 5.10-5.60(\mathrm{C}=\mathrm{CH}), 5.70-$ $6.00(\mathrm{C}=\mathrm{CH}), 6.70-8.10(\mathrm{Ph}), 8.30-9.00(\mathrm{NH})$.

Poly $\left(5^{\prime}\right) . \quad$ Yield $=63 \% . M_{\mathrm{n}}=5700, M_{\mathrm{w}} / M_{\mathrm{n}}=1.90 .[\alpha]_{\mathrm{D}}=$ $-57^{\circ}\left(c=0.10 \mathrm{~g} \cdot \mathrm{dL}^{-1}, \mathrm{CHCl}_{3}\right)$. IR $\left(\mathrm{CHCl}_{3}\right): 1742,1496$, 1454, 1357, 1261, 1096, 701, $670 \mathrm{~cm}^{-1} .{ }^{1} \mathrm{H} \mathrm{NMR}\left(\mathrm{CDCl}_{3}\right)$ : $\delta=0.78-1.62 \quad\left(\mathrm{CHCH}_{3}\right), 4.10-4.74 \quad\left(\mathrm{C}=\mathrm{CCH}_{2}\right), 4.74-5.28$ [CHCH $3, \mathrm{CHPh}_{2}$ ], 5.80-6.16 $(\mathrm{C}=\mathrm{CH}), 6.80-7.42\left(\mathrm{Ph}_{2}\right)$.

\section{RESULTS AND DISCUSSION}

\section{Polymerization}

The polymerization of 1-11 was carried out with $[\mathrm{Rh}(\mathrm{nbd}) \mathrm{Cl}]_{2}-\mathrm{Et}_{3} \mathrm{~N}$ as a catalyst in $\mathrm{CHCl}_{3}$ at $30^{\circ} \mathrm{C}$ for $24 \mathrm{~h}$. Polymers with moderate molecular weights $\left(M_{\mathrm{n}}=\right.$ 10 000-210 000) were obtained in 58-95\% yields as listed in Table I. All the polymers were soluble in $\mathrm{CHCl}_{3}$ and $\mathrm{THF}$, while insoluble in hexane and $\mathrm{MeOH}$. The structures of the polymers were determined by IR and ${ }^{1} \mathrm{H}$ NMR spectroscopies. The polymers showed no absorption peak assignable to $\mathrm{C} \equiv \mathrm{C}$ stretching vibration observed in the monomers, indicating that acetylene polymerization proceeded to give poly(1)-poly(11) as illustrated in Scheme 1. We confirmed that the polymers took a cis-stereoregular structure, because they clearly exhibited a ${ }^{1} \mathrm{H}$ NMR signal around $6 \mathrm{ppm}$ assignable to a cisvinyl proton at the main chain, similarly to rhodium-based poly $\left(N\right.$-propargylamide)s reported so far. ${ }^{6}$ The optical rotations of the polymers $\left([\alpha]_{\mathrm{D}}=-552^{\circ} \sim+74^{\circ}\right)$ measured in $\mathrm{CHCl}_{3}$ were much larger than those of the corresponding monomers $\left([\alpha]_{\mathrm{D}}=-6.4^{\circ} \sim+49^{\circ}\right)$ as similarly observed in the cases of helical polymethacrylates and polyisocyanates. ${ }^{10}$ It is suggest-

Table I. Polymerization of $1-11^{a}$

\begin{tabular}{|c|c|c|c|c|c|}
\hline \multirow{3}{*}{ Monomer } & \multicolumn{5}{|c|}{ Polymer } \\
\hline & Yield $^{b}$ & \multirow{2}{*}{$M_{\mathrm{n}}{ }^{c}$} & \multirow{2}{*}{$M_{\mathrm{w}} / M_{\mathrm{n}}{ }^{c}$} & {$[\alpha]_{D}^{d}$} & $\lambda_{\max }{ }^{e}$ \\
\hline & $\%$ & & & $\circ$ & $\mathrm{nm}$ \\
\hline 1 & 59 & 10000 & 2.00 & -153 & 302 \\
\hline 2 & 70 & 18000 & 1.70 & -104 & 308 \\
\hline 3 & 68 & 11000 & 2.41 & -452 & 312 \\
\hline 4 & 74 & 13000 & 2.22 & -290 & 319 \\
\hline 5 & 76 & 14000 & 5.66 & -355 & 333 \\
\hline 6 & 89 & 13000 & 2.68 & -307 & 342 \\
\hline 7 & 76 & 210000 & 3.00 & -552 & 385 \\
\hline 8 & 68 & 25000 & 2.22 & -290 & 319 \\
\hline 9 & 95 & 35000 & 1.64 & -201 & 314 \\
\hline 10 & 58 & 14000 & 4.32 & +74 & 279 \\
\hline 11 & 89 & 13000 & 1.85 & +20 & $-{ }^{f}$ \\
\hline
\end{tabular}

a Polymerized with $[(\mathrm{nbd}) \mathrm{RhCl}]_{2}-\mathrm{Et}_{3} \mathrm{~N}$ in $\mathrm{CHCl}_{3}$, at $30^{\circ} \mathrm{C}$ for $24 \mathrm{~h}$ $[\mathrm{M}]_{0}=1.0 \mathrm{M},[\mathrm{Rh}]=10.0 \mathrm{mM},\left[\mathrm{Et}_{3} \mathrm{~N}\right]=100 \mathrm{mM}$. ${ }^{b} \mathrm{MeOH}$-insoluble part. ${ }^{c}$ Estimated by GPC (eluent THF, PSt calibration). ${ }^{d}$ Measured by polarimetry in $\mathrm{CHCl}_{3}$ at room temperature $\left(c=0.10 \mathrm{~g} \cdot \mathrm{dL}^{-1}\right)$. ${ }^{e}$ Measured in $\mathrm{CHCl}_{3}(c=0.100 \mathrm{mM})$ at $20^{\circ} \mathrm{C} .{ }^{f}$ No peak top was observed in the range of $270-500 \mathrm{~nm}$. 


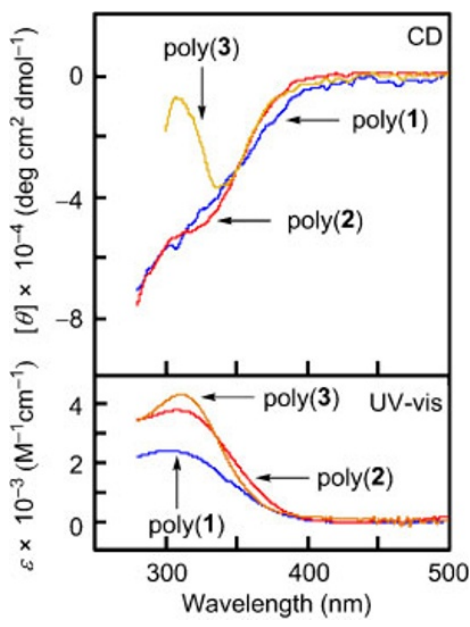

Figure 1. $\mathrm{CD}$ and UV-vis spectra of poly(1)-poly(3) measured in $\mathrm{CHCl}_{3}$ $(c=0.100 \mathrm{mM})$ at $20^{\circ} \mathrm{C}$
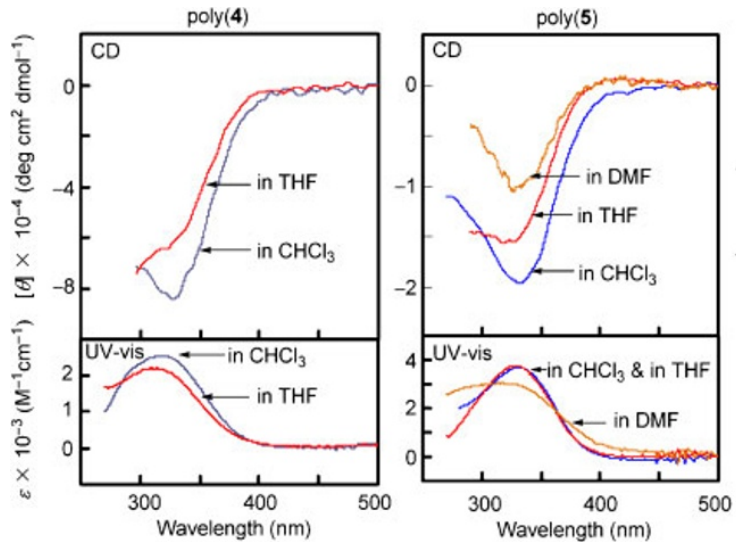

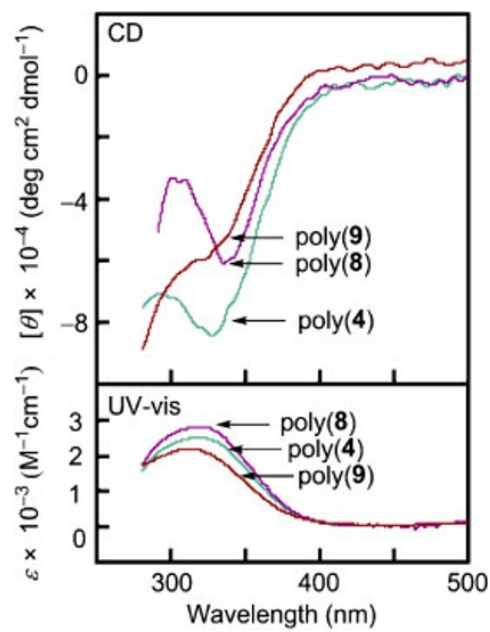

Figure 3. $\mathrm{CD}$ and UV-vis spectra of poly(4), poly(8), and poly(9) measured in $\mathrm{CHCl}_{3}(c=0.100 \mathrm{mM})$ at $20^{\circ} \mathrm{C}$.
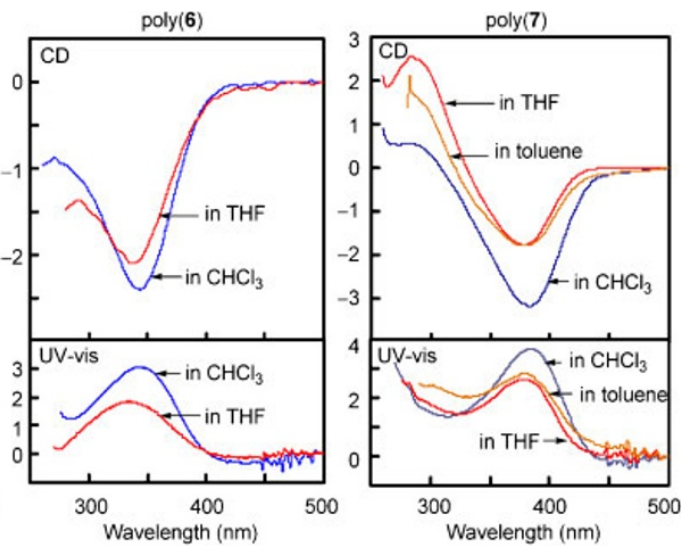

Figure 2. CD and UV-vis spectra of poly(4)-poly(7) measured in $\mathrm{CHCl}_{3}$, THF, DMF, and toluene $(c=0.100 \mathrm{mM})$ at $20^{\circ} \mathrm{C}$.

ed that the present polymers form a helical structure with predominantly one-handed screw sense.

\section{Conformation of the Polymers in Solution}

Figure 1 depicts the $\mathrm{CD}$ and $\mathrm{UV}-\mathrm{vis}$ spectra of the polymers with $\mathrm{R}^{1}=\mathrm{CH}_{3}$ and $\mathrm{R}^{2}=\mathrm{C}_{2} \mathrm{H}_{5}, n-\mathrm{C}_{4} \mathrm{H}_{9}, \mathrm{C}\left(\mathrm{CH}_{3}\right)_{3}$. Although poly $(3)$ exhibited a different $\mathrm{CD}$ pattern from those of poly(1) and poly(2), all these polymers showed UV-vis absorption at $310 \mathrm{~nm}$, indicating the absence of a substituent effect on the conjugation length.

On the other hand, poly(4)-poly(7) with $\mathrm{R}^{1}=\mathrm{CH}_{3}$ and $\mathrm{R}^{2}=\mathrm{CH}_{2} \mathrm{Ph}, \mathrm{CHPh}_{2}, \mathrm{CPh}_{3}, 1,4-\mathrm{C}_{6} \mathrm{H}_{4}-n-\mathrm{C}_{6} \mathrm{H}_{13}$ clearly exhibited a substituent effect on the UV-vis absorption wavelength. The $\lambda_{\max }$ of poly(4)-poly(6) was red-shifted in accordance with the number of phenyl group as shown in Figure 2. Comparing poly(4)-poly(6) with poly(7) bearing no spacer between the ester and benzene ring, $\operatorname{poly}(7)$ exhibited a $\lambda_{\max }$ at a longer wavelength region. A possible reason is the higher molecular weight of $\operatorname{poly}(7)\left(M_{\mathrm{n}} 210000\right)$ than those of poly(4)-poly(6) ( $\left.M_{\mathrm{n}} 13000-14000\right)$, because it is regarded that substituted polyacetylenes increase the $\lambda_{\max }$ according to the molecular weight to some extent in a manner similar to nonsubstituted polyacetylene. ${ }^{11}$ The solvent did not affect the $\lambda_{\max }$ of the polymers so much. It is considered that all these polymers have the same predominant helical sense one another, judging from the signs of $[\alpha]_{D}$ and CD signal.

Further increase in the methylene spacer length between the main chain and phenyl group did not affect the $\lambda_{\max }$ so much. As shown in Figure 3, poly(4), $\operatorname{poly}(\mathbf{8})$, and $\operatorname{poly}(\mathbf{9})$ having one, two, and three methylenes exhibited UV-vis absorption at the same wavelength $(320 \mathrm{~nm})$.

Interestingly, replacement of $\mathrm{R}^{1}$ from $\mathrm{CH}_{3}$ to $\mathrm{CH}_{2} \mathrm{Ph}$ resulted in blue-shift of UV-vis absorption of the polymers as depicted in Figure 4. Both $\lambda_{\max }$ values of poly(10) and poly(11) shorter than $300 \mathrm{~nm}$ indicate that these polymers take a tightly twisted helical conformation, ${ }^{12}$ which accompanies intramolecular hydrogen bonds between $n$th and $(n+3)$ th units. ${ }^{13}$ It seems that the $\mathrm{CH}_{2} \mathrm{Ph}$ group at the chiral center decides the helicity, and the acyl group slightly affects it.

\section{Confirmation of Hydrogen Bond}

Helical poly( $N$-propargylamide)s stabilize the conformation 

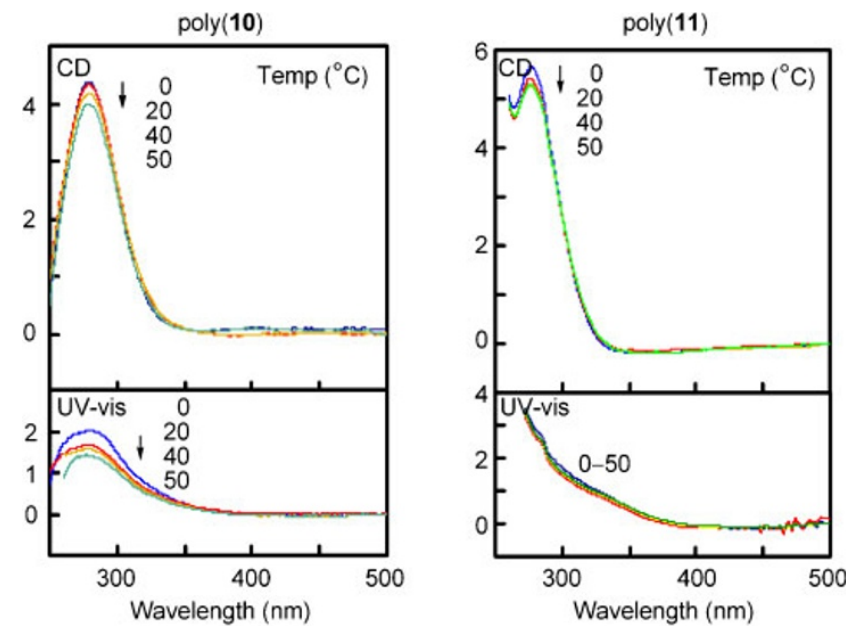

Figure 4. $C D$ and UV-vis spectra of poly(10) and poly(11) measured in $\mathrm{CHCl}_{3}(c=0.100 \mathrm{mM})$ at $0-50^{\circ} \mathrm{C}$.

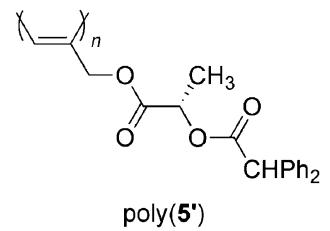

Chart 1. Structure of poly $\left(5^{\prime}\right)$

Table II. IR absorption data of $\mathbf{5}$, poly $(\mathbf{5}), \mathbf{5}^{\prime}$, and poly $\left(\mathbf{5}^{\prime}\right)^{a}$

\begin{tabular}{cccc}
\hline \multirow{2}{*}{ Compound } & \multicolumn{3}{c}{ Wavenumber $\left(\mathrm{cm}^{-1}\right)$} \\
\cline { 2 - 4 } & Ester $\mathrm{C}=\mathrm{O}$ & Amide $\mathrm{C}=\mathrm{O}$ & Amide $\mathrm{N}-\mathrm{H}$ \\
\hline $\mathbf{5}$ & 1742 & 1676 & 1521 \\
Poly(5) & 1740 & 1659 & 1542 \\
$\mathbf{5}^{\prime}$ & 1743 & - & - \\
Poly $\left(\mathbf{5}^{\prime}\right)$ & 1742 & - & - \\
\hline
\end{tabular}

${ }^{a}$ Measured in $\mathrm{CHCl}_{3}(c=5-50 \mathrm{mM})$.

by intramolecular hydrogen bonds between the amide groups as well as steric repulsion. ${ }^{6}$ To confirm the formation of hydrogen bonding, we synthesized a poly(propargyl ester) [poly $\left(\mathbf{5}^{\prime}\right)$ in Chart 1], an analogous polymer of poly(5), and compared the IR absorption of $\mathrm{C}=\mathrm{O}$ with the monomers in solution state. As listed in Table II, the absorption peaks derived from the amide $\mathrm{C}=\mathrm{O}$ and $\mathrm{N}-\mathrm{H}$ of poly $(\mathbf{5})$ were observed 17 and $21 \mathrm{~cm}^{-1}$ at lower and higher wavelengths than those of 5, respectively. On the contrary, the ester $\mathrm{C}=\mathrm{O}$ absorption peaks of poly(5) and $\mathbf{5}$ were observed at almost the same wavenumber. These values were constant irrespective of the concentration in the range of $5-50 \mathrm{mM}$. The analogous ester monomer and polymer, $\mathbf{5}^{\prime}$ and poly $\left(\mathbf{5}^{\prime}\right)$ exhibited the ester $\mathrm{C}=\mathrm{O}$ absorption peaks at the same position. Consequently, it is concluded that poly $(\mathbf{5})$ forms intramolecular hydrogen bonds between the amide groups in a manner similar to $\operatorname{poly}(N$ propargylamide)s reported so far, and the ester group does not participate in hydrogen bonding. Poly $\left(\mathbf{5}^{\prime}\right)$ also exhibited a CD signal in $\mathrm{CHCl}_{3}$ but the intensity $\left([\theta]=-3300 \mathrm{deg} \mathrm{cm}^{2} \mathrm{dmol}^{-1}\right.$ at $308 \mathrm{~nm}$ ) was negligibly small compared to that of poly $(\mathbf{5})$
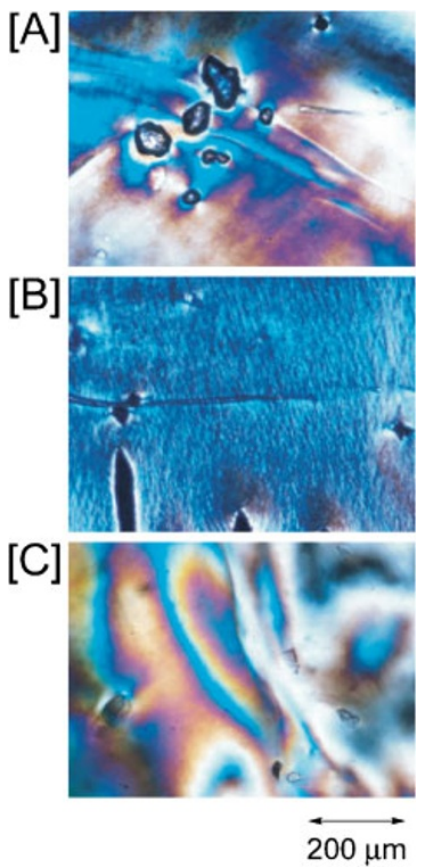

Figure 5. Polarized optical microscope images of poly $(7) ;[A]$ toluene solution $(c=30 \mathrm{wt} \%)$, [B] sheared sample, [C] THF solution $(c=30$ wt \%) measured at room temperature.

([ $\theta]=-19000 \mathrm{deg} \mathrm{cm}^{2} \mathrm{dmol}^{-1}$ at $\left.333 \mathrm{~nm}\right)$. This fact indicates that poly $\left(\mathbf{5}^{\prime}\right)$ forms no regulated structure, and the amide group is indispensable to the formation of a helical structure with predominantly one-handed screw sense.

\section{Liquid Crystalline Property}

Figure 5 depicts the polarized optical microscope images of poly(7) taken in toluene and THF solutions. As shown in [A] and $[\mathrm{C}]$ in Figure 5, the samples clearly exhibited nematic Schlieren textures, and the toluene solution exhibited a fingerprint texture after shearing (Figure $5[\mathrm{~B}]$ ), indicating the formation of a cholesteric liquid crystal. On the other hand, the other polymers exhibited no texture based on a liquid crystal. Interchain interaction between the long alkyl chains possibly plays an important role for the present polymers to induce cholesteric liquid crystalline state. The benzyl group at the chiral center of poly(11) may be unfavorable to arrangement of polymer molecules than the methyl group of poly(7), as similarly observed in the case of poly( $N$-propargylamide)s carrying azobenzene. ${ }^{13 \mathrm{a}}$

\section{CONCLUSION}

In the present study, we have demonstrated the synthesis of chiral $N$-propargylamides 1-11 from lactic acid and phenyllactic acid, and their polymerization using a rhodium catalyst. Cis-stereoregular polymers $[\operatorname{poly}(\mathbf{1})$-poly(11)] were obtained, and they formed a helical structure with predominantly onehanded screw sense. It was confirmed that the polymers form intramolecular hydrogen bonds between the amide side chains by solution state IR spectroscopic measurement. Since the 
analogous ester polymer $\left[\operatorname{poly}\left(\mathbf{5}^{\prime}\right)\right]$ did not form a helix, it was considered that the hydrogen bonds played an important role for stabilization of the helical structure. No substituent effect on the conjugation length was observed among poly(1)-poly(3) bearing $\mathrm{C}_{2} \mathrm{H}_{5}, n-\mathrm{C}_{4} \mathrm{H}_{9}$, and $\mathrm{C}\left(\mathrm{CH}_{3}\right)_{3}$ groups at the ester acyl part. Insertion of methylene spacers between the main chain and phenyl group did not affect the conjugation length of the polymers $[\operatorname{poly}(4), \operatorname{poly}(8)$, and $\operatorname{poly}(\mathbf{9})]$, either. On the other hand, poly(4)-poly(6) bearing $\mathrm{CH}_{2} \mathrm{Ph}, \mathrm{CHPh}_{2}$, and $\mathrm{CPh}_{3}$, clearly exhibited a substituent effect. The polymers bearing more phenyl groups formed looser helices; the bulky side chain made the polymer chain more flat. Replacement of the subsitituent at the chiral center from $\mathrm{CH}_{3}$ group $[\operatorname{poly}(\mathbf{1})$ and poly(7)] to $\mathrm{CH}_{2} \mathrm{Ph}$ group [poly(10) and poly(11)] resulted in tightening the helical structure. Poly(7) bearing a long alkyl chain formed a liquid crystal, while the other polymer did not, indicating that the interaction between the long alkyl groups is necessary for regulated arrangement of the polymer molecules. Thus, we could clarify the substituent effect on the helical structures of poly $(N$-propargylamde $)$ s. The principal obtained in the present study makes it possible to control the helicity and conjugation length of poly( $N$-propargylamde)s, leading to flexible tuning of optically active properties based on the helical structures.

Acknowledgment. This research was partly supported by a Grant-in-Aid for Science Research in a Priority Area "SuperHierarchical Structures (No. 446)" from the Ministry of Education, Culture, Sports, Science, and Technology, Japan. We appreciate Prof. Giseop Kwak at Kyungpook National University and Prof. Michiya Fujiki at Nara Institute of Science and Technology for optical microscopic measurement, and Prof. Junji Watanabe at Tokyo Institute of Technology for helpful discussion about the liquid crystalline property of polymers.

Received: April 12, 2008 Accepted: May 9, 2008 Published: July 2, 2008

\section{REFERENCES}

1. a) T. Masuda, J. Polym. Sci., Part A: Polym. Chem., 45, 165 (2007). b) T. Masuda, F. Sanda, and M. Shiotsuki, in: "Comprehensive Organometallic Chemistry III,” R. Crabtree, M. Mingos, Ed.,
Elsevier, Oxford 2006, vol. 11, chapter 18.

c) T. Masuda and F. Sanda, in: "Handbook of Metathesis," R. H. Grubbs, Ed., Wiley-VCH, Weinheim, 2003, vol. 3, p. 375.

d) K. Nagai, T. Masuda, T. Nakagawa, B. D. Freeman, and I. Pinnau, Prog. Polym. Sci., 26, 721 (2001).

2. M. Tabata, W. Yang, and K. Yokota, Polym. J., 22, 1105 (1990).

3. a) F. Ciardelli, S. Lanzillo, and O. Pieroni, Macromolecules, 7, 174 (1974).

b) O. Pieroni, F. Matera, and F. Ciardelli, Tetrahedron Lett., 13, 597 (1972).

4. Reviews: a) T. Aoki, T. Kaneko, and M. Teraguchi, Polymer, 47, 4867 (2006).

b) J. W. Y. Lam and B. Z. Tang, Acc. Chem. Res., 38, 745 (2005).

c) E. Yashima, K. Maeda, and T. Nishimura, Chem. Eur. J., 10, 42 (2004).

5. a) H. Nakako, R. Nomura, and T. Masuda, Macromolecules, 34, 1496 (2001).

b) R. Nomura, Y. Fukushima, H. Nakako, and T. Masuda, J. Am Chem. Soc., 122, 8830 (2000).

c) H. Nakako, Y. Mayahara, R. Nomura, M. Tabata, and T. Masuda, Macromolecules, 33, 3978 (2000).

d) H. Nakako, R. Nomura, M. Tabata, and T. Masuda, Macromolecules, 32, 2861 (1999).

6. a) J. Tabei, M. Shiotsuki, F. Sanda, and T. Masuda, Macromolecules, 38, 5860 (2005).

b) J. Tabei, R. Nomura, F. Sanda, and T. Masuda, Macromolecules, 36, 8603 (2003).

c) R. Nomura, J. Tabei, S. Nishiura, and T. Masuda, Macromolecules, 36, 561 (2003).

d) J. Tabei, R. Nomura, and T. Masuda, Macromolecules, 35, 5405 (2002).

e) R. Nomura, J. Tabei, and T. Masuda, Macromolecules, 35, 2955 (2002).

f) R. Nomura, J. Tabei, and T. Masuda, J. Am. Chem. Soc., 123, 8430 (2001).

7. a) F. Sanda, S. Nishiura, M. Shiotsuki, and T. Masuda, Macromolecules, 38, 3075 (2005).

b) R. Nomura, S. Nishiura, J. Tabei, F. Sanda, and T. Masuda, Macromolecules, 36, 5076 (2003).

8. F. Sanda, H. Araki, and T. Masuda, Macromolecules, 38, 10605 (2005).

9. F. Sanda, T. Fujii, M. Shiotsuki, and T. Masuda, Macromol. Chem. Phys., 209, 112 (2008).

10. T. Nakano and Y. Okamoto, Chem. Rev., 101, 4013 (2001).

11. I. Harada, M. Tasumi, H. Shirakawa, and S. Ikeda, Chem. Lett., 1411 (1978).

12. V. Percec, M. Obata, J. G. Rudick, B. B. De, M. Glodde, T. K. Bera, S. N. Magonov, V. S. K. Balagurusamy, and P. A. Heiney, J. Polym. Sci., Part A: Polym. Chem., 40, 3509 (2002).

13. a) T. Fujii, M. Shiotsuki, Y. Inai, F. Sanda, and T. Masuda, Macromolecules, 40, 7079 (2007).

b) F. Sanda, J. Tabei, M. Shiotsuki, and T. Masuda, Sci. Tech. Adv. Mater., 7, 572 (2006). 